\title{
Stage IIIA Primary Peritoneal Cancer AJCC v7
}

National Cancer Institute

\section{Source}

National Cancer Institute. Stage IIIA Primary Peritoneal Cancer A/CC V7. NCI Thesaurus. Code C115095.

Stage IIIA includes: T3a, N0, M0. T3a: Microscopic peritoneal tumor beyond pelvis (no macroscopic tumor). N0: No regional lymph node metastasis. M0: No distant metastasis. (AJCC 7th Ed.) 